\title{
Differential effect of surgery and radiotherapy on neurocognitive functioning and health-related quality of life in WHO grade I meningioma patients
}

\author{
David van Nieuwenhuizen · Martin Klein • \\ Lukas J. A. Stalpers $\cdot$ Sieger Leenstra $\cdot$ \\ Jan J. Heimans · Jaap C. Reijneveld
}

Received: 8 January 2007/Accepted: 23 February 2007/Published online: 13 April 2007

(C) Springer Science+Business Media B.V. 2007

\begin{abstract}
Background Potential treatment-related neurotoxicity and the indolent course of the disease mainly feed the controversy concerning the optimal timing of surgery and radiotherapy in meningioma patients.

Object To quantify the additional negative effects of conventional radiotherapy compared to surgery alone on neurocognitive functioning and health-related quality of life (HRQOL) in patients with WHO grade I meningiomas. Methods Neurocognitive functioning and HRQOL (SF36, EORTC-BCM20) were assessed in consecutive patients (1999-2005) with WHO grade I meningiomas at least 1 year after surgical treatment in two centers for brain
\end{abstract}

D. van Nieuwenhuizen · J. J. Heimans · J. C. Reijneveld ( $\square)$ Department of Neurology, VU University Medical Center, De Boelelaan 1117-1118, 1081 HV Amsterdam, The Netherlands

e-mail: JC.Reijneveld@vumc.nl

D. van Nieuwenhuizen · M. Klein

Department of Medical Psychology, VU University Medical

Center, Amsterdam, The Netherlands

\section{J. A. Stalpers}

Department of Radiotherapy, Academic Medical Center

(AMC), University of Amsterdam, Amsterdam, The Netherlands

J. C. Reijneveld

Department of Neurology, Academic Medical Center

(AMC), University of Amsterdam, Amsterdam, The Netherlands

S. Leenstra

Department of Neurosurgery, Academic Medical Center

(AMC), University of Amsterdam, Amsterdam, The Netherlands

S. Leenstra

Department of Neurosurgery, St Elizabeth Ziekenhuis, Tilburg,

The Netherlands tumor patients. Subsequently, we selected all patients who underwent surgery and conformal external beam fractioned radiotherapy $(n=18)$ and matched these patients for age, sex, and educational level with the same number of patients who had had surgery only $(n=18)$, as well as with the same number of healthy controls.

Results No significant differences in neurocognitive functioning were found between the two meningioma patient groups; however, even meningioma patients who were treated with surgery only had a significantly lower neurocognitive functioning than healthy controls. Meningioma patients who were treated with surgery and radiotherapy had significantly lower HRQOL scores than meningioma patients who were treated with surgery only, who had HRQOL ratings comparable with healthy controls; these differences, however, disappeared after correction for the duration of disease.

Conclusions In contrast with conventional thinking, longterm neurocognitive functioning was significantly impaired in our meningioma patients. Additional radiotherapy following surgery, however, does not have additional deleterious effects on neurocognitive outcome in these patients.

Keywords Meningioma - Surgery - Radiotherapy · Treatment effects - Health-related quality of life . Neurocognitive functioning
Abbreviations
HRQOL Health-related quality of life
WHO World Health Organization
RT- $\quad$ Surgery only
RT+ Surgery and adjuvant external beam conformal radiotherapy
IQ Intelligence quotient
PF Physical functioning 


$\begin{array}{ll}\text { RP } & \text { Role limitation caused by physical health } \\ & \text { problems } \\ \text { BP } & \text { Bodily pain } \\ \text { GH } & \text { General health } \\ \text { VT } & \text { Vitality } \\ \text { SF } & \text { Social functioning } \\ \text { RE } & \text { Role limitation caused by emotion problems } \\ \text { MH } & \text { Mental health } \\ \text { PCS } & \text { Physical component scale } \\ \text { MCS } & \text { Mental component scale }\end{array}$

\section{Introduction}

Meningiomas (i.e. primary tumors arising from the dural coverings of the brain) are the most common primary non-glial brain tumors, accounting for $13-26 \%$ of all primary brain tumors. Meningiomas have an annual incidence of approximately 6 per 100,000 population [1]. Histological grading of meningiomas is based upon the current World Health Organization (WHO) classification [2]. About $90 \%$ of the meningiomas are benign, which corresponds to WHO grade I. Atypical meningiomas (WHO grade II) make up 5-7\%, and anaplastic variants (WHO grade III) arise in $1-3 \%$ of the cases [3].

Surgical excision of the tumor and its dural base is the usual initial treatment, particularly in easily accessible tumors localized on the outer brain surface or at the cerebral falx between both hemispheres (so-called convexity tumors). Radiotherapy has clinical benefits when tumor histology reveals atypia or anaplasia (WHO grade II and III). Radiotherapy also reduces the risk of local recurrence of grade I tumors after a subtotal resection. However, the clinical value and optimal timing of radiotherapy after (in-) complete surgery or after recurrence remains a matter of debate [4-7].

The controversy on the choice and timing of radiotherapy can be attributed to a lack of knowledge regarding its side effects. The few available data indicate that about $30 \%$ of meningioma patients experience severe long-term cerebral sequelae, mostly neurocognitive deficits [8-10]. It is unclear, however, whether this is due to pre-treatment brain damage by the tumor itself, to surgery, to radiotherapy, or to a combination of these. For other types of primary brain tumors it is known that neurocognitive deficits in patients can be attributed to a combination of these factors $[11,12]$. Complications of treatment, especially when resulting in neurocognitive dysfunction, have a great impact on patients and their proxies. Unfortunately, little is known about the effect of different treatment options on the frequency and severity of neurocognitive dysfunction in meningioma patients. More information on this issue will lead to improved understanding of the complaints of meningioma patients, and offer support in the choice and timing of treatment.

The present study aimed to determine the effect of additional radiotherapy on neurocognitive functioning. We hypothesized that additional radiotherapy in meningioma patients will have a cumulative negative effect on neurocognitive functioning and health-related quality of life (HRQOL) compared with surgery only. To put neurocognitive functioning and HRQOL of meningioma patients who underwent surgery only in perspective, we compared their results with normative data from healthy general population samples.

\section{Patients and methods}

\section{Patients}

This study is part of a two-center, retrospective investigation into the prevalence and severity of neurocognitive problems in meningioma patients. For the present study we interviewed all adult ( $>18$ years) patients with WHO grade I intracranial meningiomas, who were treated either with surgery only (RT-), or with surgery and adjuvant external beam conformal radiotherapy ( $\mathrm{RT}+$ ) at two tertiary referral centers for brain tumor patients in Amsterdam (i.e. the Academic Medical Center and the VU University Medical Center) from 1999 through 2005. In these centers, the decision to treat meningioma patients with radiotherapy after surgery is always made on basis of localization and size of the tumor. In some patients the tumor could only be resected partially because of the localization of the tumor, mainly skullbase meningiomas. In these patients and in patients with a recurrence after one or more surgical resections, additional radiotherapy was given. Patients must have undergone their last treatment at least 1 year previously. Exclusion criteria were: treatment by stereotactic radiotherapy, or the presence of two or more of the following conditions: cerebrovascular pathology, presence of other tumors of the nervous system, congenital malformations of the nervous system, multiple sclerosis, Parkinson's disease, organic psychosis (other than dementia), and schizophrenia. Also excluded were patients with optic nerve meningiomas. Patients had to have sufficient command of the Dutch language to be able to carry out the neurocognitive tests. The medical ethics committees of both medical centers approved the study protocol. Eligibility was checked by medical chart review and, if necessary, with the general practitioner.

A total 89 patients with intracranial WHO grade I meningiomas were recruited, of whom 61 (69\%) 
underwent surgery, 4 received radiotherapy only (4\%), 21 had surgery and adjuvant conformal external beam radiotherapy $(24 \%)$ and 3 received neither surgical treatment nor radiotherapy (3\%). We invited patients by letter. Informed consent procedures preceded patients' agreement to participate. Eventually, 11 eligible patients declined to participate; of these patients, 6 underwent surgery, 3 underwent surgery and subsequent radiotherapy, and 2 had not received therapy. The main reason for refusal was that participation was too burdensome. In total $94 \%$ of the meningioma patients who underwent RT- and $83 \%$ of the $\mathrm{RT}+$ patients were tested at home; the remaining patients were tested in the hospital. Clinical data obtained from medical chart review at entry, included tumor characteristics [histology, location (convexity, tentorium/falx, skull base, orbit), size, hyperostosis, and edema]. The preoperative tumor volume was estimated by assuming an ellipsoid of the orthogonal tumor diameters $x, y$, and $z$ on CT-scan and/or MRI:

Tumor volume $=4 / 3 \pi *(1 / 2 x * 1 / 2 y * 1 / 2 z)$

Tumor area $=\pi *(1 / 2 x * 1 / 2 y)$

Before neuropsychological testing, the patients completed a questionnaire regarding sociodemographic data (including age, sex, and educational level) and a questionnaire on HRQOL, and epilepsy and its treatment [13].

For this study, we selected all patients from the database who underwent surgery and adjuvant radiotherapy (RT+) and matched these patients with the same number of patients from the database who underwent surgery only. Patients were matched for age, sex, and educational level.

\section{Healthy controls}

In addition to RT- and RT+ patients, normative data of healthy controls were used as an additional anchor to interpret the results. Healthy controls were drawn from a large, cross-sectional study of the biological and psychological determinants of neurocognitive aging, the Maastricht Aging study [14]. We matched this control group with RT- patients with respect to age, sex, and educational level. Educational level was assessed by a Dutch scoring system consisting of an eight-point scale, ranging from unfinished primary education (level 1) to university education (level 8). In order to compare HRQOL outcomes, healthy controls matched for age, sex, and educational level were drawn from a nationwide study that aimed to translate, validate, and generate normative data on the Short-Form Health Survey (SF-36) for use among Dutch-speaking residents of the Netherlands [15].

\section{Study measures}

Health-related quality of life

We assessed patients' overall degree of physical function with the Karnofsky Performance Status (KPS) scale, which is frequently used in clinical cancer research. Scores range from 0 (lowest score) to 100 (highest level) [16]. The ability to perform daily activities was assessed with the Barthel Activities of Daily Living index [17]. The index consists of ten items (assessing continence of bowel and bladder, grooming, toilet use, feeding, transfer, mobility, dressing, climbing stairs, and bathing); higher scores indicate good functional independence.

Neurological functioning was scored with the neurological functioning scale developed by Order et al. [18]. Scores for this scale range from 1 to 4 , with higher scores indicating intact neurological functioning. For self-reported HRQOL we used the MOS Short-Form Health Survey (SF-36) [19]. The SF-36 is composed of 36 items, organized into eight multi-item scales assessing physical functioning (PF), role limitation caused by physical health problems (RP), bodily pain (BP), general health $(\mathrm{GH})$, vitality (VT), social functioning (SF), role limitation caused by emotional problems (RE), and mental health $(\mathrm{MH})$. Rough scores are converted linearly to $0-100$ scales, with higher scores representing better levels of functioning. In addition, we calculated two higher-order compound scores, a physical component scale (PCS) and a mental component scale (MCS). The BCM-20 questionnaire was used to assess additional health problems associated specifically with meningioma and its treatment [20]. Of the $20 \mathrm{BCM}$ items, 13 are organized into 5 subscales assessing future uncertainty, visual disorder, motor dysfunction, communication deficit, and emotional distress. The remaining seven items assess other disease symptoms and side-effects of treatment prevalent among patients with brain tumors, including headaches, seizures, drowsiness, hair loss, itching, weakness of the legs, and lack of bladder control. Since emotional status was already assessed by the SF-36, the 4-item emotional distress scale of the BCM20 was not analyzed.

Neurocognitive functioning

Because of the different causes and severity of neurocognitive problems, we used a wide range of tests to assess neurocognitive functions. Neurocognitive functions refer to an individual's ability to perceive, store, retrieve, and use sensory and perceptual information from the environment and past experience, and to such mental activities as planning and organizing. A battery of standard tests was 
used to assess neuropsychological status. The total time required to complete the battery was approximately $60 \mathrm{~min}$. Appendix 1 provides detailed information on this test battery.

\section{Statistical analysis}

Statistical analyses were performed with SPSS (version 11.0). Chi-square tests were used to match the RT-patients with healthy controls for sex. Chi-square tests were also used to show differences in pathological features (e.g. meningothelial, transitional) between RT- and RT+ patients. RT+ meningioma patients were compared with RT- meningioma patients, and RT- meningioma patients were compared with healthy controls for HRQOL and neurocognitive functioning. Student's $t$ test was used for independent samples to determine whether neurocognitive function and HRQOL of meningioma RT+ patients differed from that of meningioma RT- patients. Student's $t$ test was also used to determine whether neurocognitive functioning and HRQOL of RT- meningioma patients differed from that of healthy controls. The level of significance was set at $P<0.05$.

\section{Results}

\section{Patients' characteristics}

Included were 18 patients who underwent surgery followed by radiotherapy $(\mathrm{RT}+)$ and these patients were matched with 18 patients who underwent surgery only (RT-). Table 1 presents the sociodemographic characteristics of the RT- and the RT+ patients. Regarding the RT+ patients, adjuvant radiotherapy consisted of conformal external beam fractionated radiotherapy; the radiation dose was 50.4-54.0 Gy in 1.8-2.0 Gy per fraction, five fractions per week, using 6-10 MeV photon beams. None of the 36 patients had clinical or radiological signs of tumor progression.

The near optimal levels for neurological functioning (Order scale) and daily living (Barthel index) did not differ significantly between the two groups, although RT+ meningioma patients were significantly more limited in their physical functioning (PCS) than RT- patients. In RTmeningioma patients, more tumors were localized in the convexity and less at the skull base than in RT+ meningioma patients. There were no significant differences in tumor volume, nor in pathology subgroups, between RTand RT+ patients. Although, follow-up time was significantly different between RT- and RT+ patients, no differences were seen in time since last treatment.
Table 1 Sociodemographic and clinical characteristics of the study patients

\begin{tabular}{|c|c|c|c|c|c|}
\hline \multirow{2}{*}{$\begin{array}{l}\text { Patient groups } \\
\text { Variables }\end{array}$} & \multicolumn{2}{|c|}{$\begin{array}{l}\text { Surgery only } \\
(n=18)\end{array}$} & \multicolumn{3}{|c|}{$\begin{array}{l}\text { Surgery plus } \\
\text { radiotherapy }(n=18)\end{array}$} \\
\hline & $M$ & SD & $M$ & SD & $P^{*}$ \\
\hline \multicolumn{6}{|l|}{ Characteristics } \\
\hline Mean age in years & 62.6 & 11.8 & 63.3 & 10.6 & 0.424 \\
\hline Male:female ratio & $1: 7$ & $1: 8$ & & & \\
\hline Educational level $^{\mathrm{a}}$ & 3.28 & 2.1 & 3.17 & 1.3 & 0.425 \\
\hline Mean IQ & 99.2 & 17.4 & 95.1 & 16.0 & 0.236 \\
\hline Years since diagnosis $^{\mathrm{a}}$ & 3.0 & 1.7 & 7.6 & 6.3 & 0.013 \\
\hline Years since last treatment ${ }^{\mathrm{a}}$ & 3.3 & 2.0 & 3.3 & 1.9 & 0.982 \\
\hline \multicolumn{6}{|c|}{ Functional/performance status } \\
\hline Karnofsky $^{\mathrm{a}}$ & 82.8 & 19.6 & 71.1 & 18.4 & $\mathbf{0 . 0 3 8}$ \\
\hline Barthel $^{\mathrm{a}}$ & 17.2 & 1.4 & 17.2 & 1.6 & 0.408 \\
\hline Order $^{\mathrm{a}}$ & 3.7 & 0.5 & 3.8 & 0.4 & 0.131 \\
\hline Number of tumors ${ }^{\mathrm{a}}$ & 1.0 & 0.0 & 1.5 & 1.2 & \\
\hline Volume of tumor $\left(\mathrm{ml}^{3}\right)^{\mathrm{a}}$ & 23.5 & 19.3 & 39.4 & 43.5 & 0.202 \\
\hline Area of tumor $\left(\mathrm{cm}^{2}\right)^{\mathrm{a}}$ & 11.0 & 6.8 & 15.3 & 12.0 & 0.230 \\
\hline \multicolumn{6}{|l|}{ Pathologic features } \\
\hline Meningothelial & 6 & & 9 & & 0.310 \\
\hline Fibroblastic & & & 1 & & 0.310 \\
\hline Transitional & & & 2 & & 0.146 \\
\hline Not further classified & 10 & & 6 & & 0.180 \\
\hline Unknown & 2 & & 0.146 & & \\
\hline
\end{tabular}

${ }^{a}$ Mean test scores

Neurocognitive functioning

Data on neurocognitive functioning and HRQOL are given in Tables 2, 3 and 4. RT+ patients did not have a significantly impaired performance on the Line Bisection test compared to RT- patients, which excludes a major midline deviation (Table 4). In the tests for memory, especially AVLT total recall, AVLT max and AVLT delayed recall, RT - scored significantly worse compared with healthy controls. RT+ meningioma patients did not score differently from RT- meningioma patients on most tests measuring attention and executive functioning. RT+ meningioma patients were slightly faster on Stroop card I and II, but slower on Stroop card III. For RT-meningioma patients no clear differences were seen, except that Stroop card II took more time compared with healthy controls. $\mathrm{RT}+$ patients performed worse on the Fluency test. RT+ patients took less time to accomplish the CST A and CST B test but took more time on the CST C test compared with RT - meningioma patients. RT- meningioma patients needed more time to complete for the CST tests compared with healthy controls. 
Table 2 Scores on the HRQOL test of RT- meningioma patients, RT+ meningioma patients, and healthy controls

\begin{tabular}{|c|c|c|c|c|c|c|c|c|}
\hline \multirow[t]{2}{*}{ Variables } & \multicolumn{2}{|c|}{ RT- patients $(n=18)$} & \multicolumn{3}{|c|}{$\mathrm{RT}+$ patients $(n=18)$} & \multicolumn{3}{|c|}{ Healthy controls $(n=18)$} \\
\hline & $M$ & SD & $M$ & SD & $P^{*}$ & $M$ & SD & $P^{*}$ \\
\hline Physical functioning (PF) & 72.5 & 32.7 & 54.7 & 54.47 & 0.047 & 73.9 & 25.2 & 0.381 \\
\hline Role physical (RP) & 61.1 & 43.2 & 33.8 & 38.5 & 0.032 & 63.2 & 34.4 & 0.476 \\
\hline Bodily pain (BP) & 66.6 & 40.1 & 56.3 & 23.9 & 0.184 & 61.9 & 24.6 & 0.281 \\
\hline General health $(\mathrm{GH})$ & 60.7 & 25.0 & 45.2 & 27.2 & 0.050 & 64.8 & 17.8 & 0.294 \\
\hline Vitality (VT) & 60.9 & 31.3 & 44.4 & 21.0 & 0.039 & 61.7 & 18.0 & 0.442 \\
\hline Social functioning (SF) & 69.5 & 33.8 & 66.7 & 24.3 & 0.388 & 70.6 & 25.7 & 0.472 \\
\hline Role emotional (RE) & 77.8 & 41.1 & 72.5 & 42.9 & 0.364 & 75.0 & 35.5 & 0.314 \\
\hline Mental health (MH) & 71.6 & 23.8 & 70.8 & 22.7 & 0.461 & 69.6 & 19.3 & 0.468 \\
\hline PCS & 44.8 & 13.2 & 33.2 & 11.0 & 0.007 & 45.4 & 11.6 & 0.335 \\
\hline MCS & 51.8 & 11.6 & 50.9 & 13.0 & 0.421 & 48.7 & 12.2 & 0.323 \\
\hline
\end{tabular}

Data are mean (M) and standard deviation (SD)

PCS physical component score, MCS mental component score

Health-related quality of life

Compared with RT- patients, RT+ meningioma patients scored less well on self-reported HRQOL. RT+ meningioma patients had significantly impaired physical functioning (PF), more role limitations caused by physical health problems (RP), lower Vitality (VT), and lower scores on the Physical Component Scale (PCS). These differences, however, disappeared after correction for the duration of disease. When RT- patients were compared with healthy controls, no significant differences were seen. Scores of the BCM-20 showed no significant differences between RT- and RT+ meningioma patients (Table 3).

\section{Discussion}

In this study, no significant differences were found in neurocognitive functioning between WHO grade I meningioma patients that underwent surgery only, and patients that received additional radiotherapy; however, even patients who were only treated surgically had a significantly lower neurocognitive functioning than healthy controls. The most profound neurocognitive disturbances were seen in memory tasks. Meningioma patients who were treated with surgery and radiotherapy had significantly lower HRQOL scores than meningioma patients who were treated surgically only, who had HRQOL scores comparable with healthy controls; these differences, however, disappeared after correction for the duration of disease.

Very few studies have been published on neurocognitive functioning of meningioma patients. Tucha et al. [21] examined neurocognitive functioning before and shortly
Table 3 Scores on the BCM-20 test of RT- meningioma patients, $\mathrm{RT}+$ meningioma patients

\begin{tabular}{|c|c|c|c|c|c|}
\hline \multirow[t]{2}{*}{ Variables } & \multicolumn{2}{|c|}{$\begin{array}{l}\text { RT- patients } \\
(n=18)\end{array}$} & \multicolumn{3}{|c|}{$\begin{array}{l}\mathrm{RT}+\text { patients } \\
(n=18)\end{array}$} \\
\hline & $M$ & SD & $M$ & SD & $P^{*}$ \\
\hline Future uncertainty & 23.4 & 26.2 & 28.2 & 25.9 & 0.248 \\
\hline Visual disorder & 15.0 & 22.2 & 28.4 & 28.3 & 0.065 \\
\hline Motor dysfunction & 17.0 & 26.1 & 27.2 & 25.3 & 0.126 \\
\hline Communication deficit & 20.9 & 30.4 & 19.1 & 21.2 & 0.421 \\
\hline Headaches & 23.5 & 22.9 & 40.7 & 42.1 & 0.073 \\
\hline Seizures & 5.9 & 17.6 & 13.0 & 28.3 & 0.192 \\
\hline Drowsiness & 21.6 & 31.0 & 33.3 & 32.3 & 0.141 \\
\hline Bothered by hair loss & 5.9 & 13.1 & 14.8 & 26.1 & 0.107 \\
\hline Bothered by itching skin & 23.5 & 34.9 & 24.1 & 37.6 & 0.433 \\
\hline Weakness of legs & 11.8 & 23.4 & 24.1 & 33.9 & 0.112 \\
\hline Difficulty controlling bladder & 13.7 & 23.7 & 18.5 & 32.8 & 0.313 \\
\hline
\end{tabular}

Data are mean $(\mathrm{M})$ and standard deviation $(\mathrm{SD})$

after surgery for a frontal meningioma. Surgery improved neurocognitive functioning but, compared with healthy controls, significant postoperative neurocognitive deficits remained, particularly a lowered attention span and decreased executive functions. These latter data agree with our long-term results at least 1 year after treatment. Unlike differences in histology and biology of meningioma, some striking results are similar to those reported in previous studies in patients with other types of primary brain tumors $[22,23]$. We found neurocognitive disturbances in our meningioma patients similar to those in glioma patients and, similarly, radiotherapy was not associated with poorer neurocognitive outcome. 
Table 4 Scores on the neuropsychological tests of RT- meningioma patients, RT+ meningioma patients, and healthy controls

\begin{tabular}{|c|c|c|c|c|c|c|c|c|}
\hline \multirow[t]{2}{*}{ Variables } & \multicolumn{2}{|c|}{ RT- patients $(n=18)$} & \multicolumn{3}{|c|}{$\mathrm{RT}+$ patients $(n=18)$} & \multicolumn{3}{|c|}{ Healthy RT- controls $(n=18)$} \\
\hline & $M$ & SD & $M$ & SD & $P^{*}$ & M & SD & $P^{* *}$ \\
\hline \multicolumn{9}{|l|}{ Perception } \\
\hline Line bisection-HD & 0.12 & 2.0 & 0.38 & 2.3 & 0.358 & $5.5 \%{ }^{\mathrm{a}}$ & b & b \\
\hline Line bisection-VD & 2.46 & 1.5 & 1.42 & 2.2 & 0.058 & $27.8 \%{ }^{\mathrm{a}}$ & $\mathrm{b}$ & $\mathrm{b}$ \\
\hline \multicolumn{9}{|l|}{ Memory } \\
\hline AVLT trial 1 (nc) & 4.2 & 2.2 & 4.2 & 1.5 & 0.500 & 4.4 & 2.4 & 0.414 \\
\hline AVLT total recall (nc) & 33.1 & 13.0 & 30.7 & 10.7 & 0.253 & 42.0 & 11.8 & 0.019 \\
\hline AVLT delayed recall $(\mathrm{nc})$ & 6.7 & 4.0 & 6.1 & 2.8 & 0.232 & 9.0 & 3.2 & 0.037 \\
\hline AVLT delayed recognition & 13.1 & 2.2 & 13.2 & 1.8 & 0.467 & 13.9 & 2.0 & 0.137 \\
\hline AVLT delta score & 4.8 & 2.0 & 4.1 & 2.2 & 0.135 & 6.6 & 2.5 & 0.012 \\
\hline AVLT max (nc) & 9.1 & 3.4 & 8.3 & 2.7 & 0.227 & 11.0 & 2.7 & 0.019 \\
\hline WMT slope & 17.2 & 9.5 & 16.1 & 10.9 & 0.378 & 15.2 & 5.6 & 0.223 \\
\hline WMT intercept & 33.6 & 14.3 & 39.9 & 17.8 & 0.133 & 27.5 & 6.5 & 0.056 \\
\hline \multicolumn{9}{|c|}{ Attention and executive function } \\
\hline SCWT card I (s) & 55.1 & 21.8 & 50.9 & 17.1 & 0.268 & 53.1 & 8.5 & 0.360 \\
\hline SCWT card II (s) & 72.3 & 28.4 & 71.2 & 14.8 & 0.446 & 64.0 & 9.0 & 0.121 \\
\hline SCWT card III (s) & 111.8 & 56.0 & 124.7 & 42.0 & 0.230 & 110.8 & 22.2 & 0.270 \\
\hline SCWT interference $(\%)$ & 86.7 & 62.3 & 95.7 & 37.1 & 0.311 & 89.5 & 29.6 & 0.234 \\
\hline Fluency (nc) & 20.7 & 8.9 & 18.9 & 6.2 & 0.246 & 22.3 & 5.7 & 0.269 \\
\hline CST A (s) & 38.8 & 39.0 & 31.6 & 10.0 & 0.235 & 23.1 & 5.2 & 0.051 \\
\hline CST B (s) & 50.7 & 71.5 & 40.7 & 15.7 & 0.287 & 24.5 & 5.1 & 0.065 \\
\hline CST C (s) & 53.2 & 43.6 & 55.8 & 26.5 & 0.417 & 45.0 & 21.6 & 0.162 \\
\hline Motor component (s) & 9.4 & 9.5 & 9.2 & 4.3 & 0.475 & 6.2 & 1.5 & 0.087 \\
\hline
\end{tabular}

Means (M) and standard deviations (SD) represent estimated means based on corrections for age and education for RT-and RT+ patients. Means and standard deviations for healthy control are observed scores

* $P$-values of univariate $F$ tests for RT- and RT+ patients corrected for age and educational differences

** $P$-values of $t$ tests comparisons between RT-patients and healthy age, sex, and education matched controls

${ }^{a}$ Percentage of meningioma patients who deviated $>2$ SD of healthy controls

b Age, sex, and education matched data unavailable

Despite major neurocognitive deficits, we found no impaired HRQOL in patients who had surgery only. Discrepancies between HRQOL and neurocognitive functioning have been described for other patient groups $[24,25]$. There may be limitations in the ability of patients with brain disease and resulting cognitive disturbance to appraise their own situation.

In contrast, we did find a decreased HRQOL in patients who had surgery plus radiotherapy, particularly in the physical component of the SF-36. It may be tempting to attribute the lower physical performance and quality of life to progressive radiation damage in these patients. However, the reverse is more plausible, namely that patients needing adjuvant radiotherapy had larger and more complex meningiomas that inherently cause more cerebral damage, perhaps even aggravated by more extensive surgery. Furthermore, we should keep in mind that the impending threat of tumor recurrence and heavier treatment imposes a psychological burden with resultant anxiety, depression, or fatigue, which can also negatively affect the patient's neurocognitive function [22]. Patients who had surgery only had a much shorter disease history (on average 3.0 years) than patients who had surgery plus radiotherapy (on average 7.6 years), frequently after repeated earlier surgery. After correction for time after primary diagnosis (post hoc analysis), no significant differences were seen for HRQOL between RT- and RT+ meningioma patients. Most likely, the impaired HRQOL is therefore associated with a longer disease history.

Several limitations of this study should be mentioned. First of all, because we used a retrospective design, we lack a baseline pretreatment assessment. It is possible that the $\mathrm{RT}+$ patients were functioning at a much higher HRQOL level before radiotherapy compared to the RT- patients. This would imply that the outcomes of post-treatment HRQOL should be seen in a different perspective. However, as the decision to add radiotherapy was solely based on surgical grounds, a selection bias on the basis of 
pre-existent HRQOL level is very unlikely. The anatomical distribution of meningiomas differs between both patient groups. As differences in localization might be associated with differences in vulnerability for the neurocognitive side effects of radiotherapy, e.g. higher risks for tumors involving eloquent brain areas, this should be taken into account when interpreting the neurocognitive scores of both groups. The historical cohort study design may have resulted in a selection bias and confounding bias. For example, patients with severely debilitating disease that precluded testing were excluded from analyses. Also, the number of patients is relatively small which might have influenced the results. On the other hand, this small number enabled to correct for tumor localization and use of antiepileptic drugs, factors that are known to influence neurocognitive functioning [22].

In conclusion, the results of our study strongly suggest that the addition of radiotherapy has no significant detrimental impact on late neurocognitive functioning in meningioma patients. Our data also indicate that the negative effects on cognition are due to the tumor itself or to surgery. Further study with a prospective study design including baseline scores for QOL and neurocognitive functioning will be necessary to draw definite conclusions regarding the extent and causality of neurocognitive disturbances in meningioma patients.

Acknowledgments The authors thank the students Miriam Wumkes, Martin Waagemans, Sarah Brantjes and Monica Dijkstra for their assistance in data collection and analysis. In addition, we thank Clemens M.F. Dirven, MD, PhD, (neurosurgeon at the VU University Medical Center), Frank J. Lagerwaard, MD, PhD (radiation oncologist at the VU University Medical Center) and W. Peter Vandertop, $\mathrm{MD}, \mathrm{PhD}$ (neurosurgeon at the VU University Medical Center and Academic Medical Center) for their invaluable help and critical comments.

\section{Appendix 1: Description of the neuropsychological test battery}

\section{Test domains}

overall cognitive performance

Intelligence

The Dutch Adult Reading Test (DART) [26]. The Dutch version of the New Adult Reading Test provides a measure of premorbid capacity based on verbal ability.

\section{Perception}

Line Bisection Test [27]. This test is a device for measuring unilateral neglect, which is usually a sequel of massive right hemisphere lesions. Noticeable errors are most often made by patients with visual field defects who tend to underestimate the side of the line opposite to the defective field. Outcome measures are horizontal and vertical deviations.

мemory continued

Auditory Verbal Learning Test (AVLT) [28]. This version of the Rey Auditory Verbal Learning Test calls for various aspects of verbal learning and recall. Measures used for analysis are: memory performance on trial 1 as indicator of immediate recall, total recall after five trials, delayed recall and recognition after $20 \mathrm{~min}$ as indicators of memory consolidation into long-term memory, and a delta score as a measure of learning capacity.

Working Memory Task (WMT) [29]. This task is designed to measure the speed of memory processes. The underlying principle is that the extra time needed to complete a test in which there is a stepwise increase in the amount of information to be kept in memory, is a measure of the ease at which information is processed in working memory. Capacity is measured by using the slope and intercept as a function of the number of letters to be kept in working memory.

Attention and executive function:

Stroop Color-Word Test (SCWT) [28]. This test is a selective attention task aiming at measuring interference susceptibility and consists of three subtasks with increasing task complexity.

Categoric Word Fluency [30]. This is a task requiring the generation of words from specific semantic categories (animals) within a limited time.

Concept Shifting Test (CST) [31]. This test, which has two conditions of complexity, predominantly measures functions associated with executive function, especially visual scanning and conceptual tracking. The motor component of this task is measured by three dummy conditions in which no neurocognitive capacity except for graphomotor speed is required.

\section{References}

1. Whittle IR, Smith C, Navoo P, Collie D (2004) Meningiomas. Lancet 363(9420):1535-1543

2. Kleihues P, Louis DN, Scheithauer BW, Rorke LB, Reifenberger G, Burger PC et al (2002) The WHO classification of tumors of the nervous system. J Neuropathol Exp Neurol 61(3):215-225, discussion 226-219

3. Louis DN, Scheithauer BW, Budka H, von Deimling A, Kepes JJ (2000) Meningiomas. In: Kleihues P, Cavenee WK (Eds) World Health Organization Classification of tumours. Pathology and genetics of tumors of the nervous system. IARC Press, Lyon, pp 176-184

4. Barbaro NM, Gutin PH, Wilson CB, Sheline GE, Boldrey EB, Wara WM (1987) Radiation therapy in the treatment of partially resected meningiomas. Neurosurgery 20(4):525-528

5. Goldsmith BJ, Wara WM, Wilson CB, Larson DA (1994) Postoperative irradiation for subtotally resected meningiomas. A retrospective analysis of 140 patients treated from 1967 to 1990. J Neurosurg 80(2):195-201

6. Kokubo M, Shibamoto Y, Takahashi JA, Sasai K, Oya N, Hashimoto $\mathrm{N}$ et al (2000) Efficacy of conventional radiotherapy for recurrent meningioma. J Neurooncol 48(1):51-55

7. Jaaskelainen J (1986) Seemingly complete removal of histologically benign intracranial meningioma: late recurrence rate and factors predicting recurrence in 657 patients. A multivariate analysis. Surg Neurol 26(5):461-469

8. Mathiesen T, Kihlstrom L, Karlsson B, Lindquist C (2003) Potential complications following radiotherapy for meningiomas. Surg Neurol 60(3):193-198, discussion 199-200 
9. Duchstein S, Gademann G, Peters B (2003) Early and late effects of local high dose radiotherapy of the brain on memory and attention. Strahlenther Onkol 179(7):441-451

10. Dufour H, Muracciole X, Metellus P, Regis J, Chinot O, Grisoli F (2001) Long-term tumor control and functional outcome in patients with cavernous sinus meningiomas treated by radiotherapy with or without previous surgery: is there an alternative to aggressive tumor removal? Neurosurgery 48(2):285-294, discussion 294-286

11. Klein M, Engelberts NH, van der Ploeg HM, Kasteleijn-Nolst Trenite DG, Aaronson NK, Taphoorn MJ et al (2003) Epilepsy in low-grade gliomas: the impact on cognitive function and quality of life. Ann Neurol 54(4):514-520

12. Taphoorn MJB, Klein M (2004) Cognitive deficits in adult patients with brain tumors. Lancet Neurol 3(3):159-168

13. Masmoudi K, Gras-Champel V, Masson H, Andrejak M (2006) Parkinsonism and/or cognitive impairment with valproic acid therapy: a report of ten cases. Pharmacopsychiatry 39(1):9-12

14. Jolles J, van Boxtel MP, Ponds RW, Metsemakers JF, Houx PJ (1998) The Maastricht aging study (MAAS). The longitudinal perspective of cognitive aging. Tijdschr Gerontol Geriatr 29(3):120-129

15. Aaronson NK, Muller M, Cohen PD, Essink-Bot ML, Fekkes M, Sanderman R et al (1998) Translation, validation, and norming of the Dutch language version of the SF-36 Health Survey in community and chronic disease populations. J Clin Epidemiol 51(11):1055-1068

16. Karnofsky DA, Abelmann WH, Craver LF (1948) The use of nitrogen mustards in the palliative treatment of carcinoma. Cancer 1:634-656

17. Wade DT, Collin C (1988) The Barthel ADL Index: a standard measure of physical disability? Int Disabil Stud 10(2):64-67

18. Order S, Hellman S, Von Essen C et al (1968) Improvement in quality of survival following whole-brain irridiation for brain metastasis. Radiology 91:149-153

19. Ware JE, Sherbourne CD (1992) The MOS 36-item short-form health survey (SF-36): I. Conceptual framework and item selection. Med Care 30(6):473-483
20. Osoba D, Aaronson NK, Muller M, Sneeuw K, Hsu MA, Yung WK et al (1996) The development and psychometric validation of a brain cancer quality-of-life questionnaire for use in combination with general cancer-specific questionnaires. Qual Life Res 5(1):139-150

21. Tucha O, Smely C, Preier M, Becker G, Paul GM, Lange KW (2003) Preoperative and postoperative cognitive functioning in patients with frontal meningiomas. J Neurosurg 98(1):21-31

22. Klein M, Heimans JJ, Aaronson NK, van der Ploeg HM, Grit J, Muller M et al (2002) Effect of radiotherapy and other treatmentrelated factors on mid-term to long-term cognitive sequelae in low-grade gliomas: a comparative study. Lancet 360(9343): 1361-1368

23. Laack NN, Brown PD (2004) Cognitive sequelae of brain radiation in adults. Semin Oncol 31(5):702-713

24. Giovagnoli AR (1999) Quality of life in patients with stable disease after surgery, radiotherapy, and chemotherapy for malignant brain tumour. J Neurol Neurosurg Psychiatry 67(3): 358-363

25. Spector A, Orrell M (2006) Quality of life (QoL) in dementia: a comparison of the perceptions of people with dementia and care staff in residential homes. Alzheimer Dis Assoc Disord 20(3): 160-165

26. Schmand B, Bakker D, Saan R, Louman J (1991) The dutch reading test for adults: a measure of premorbid intelligence level. Tijdschr Gerontol Geriatr 22(1):15-19

27. Schenkenberg T, Bradford DC, Ajax ET (1980) Line bisection and unilateral visual neglect in patients with neurologic impairment. Neurology 30:509-517

28. Lezak MD (2004) Neuropsychological assessment, 4th edn. Oxford University Press, New York

29. Sternberg S (1975) Memory scanning: new findings and current controversies. Q J Exp Psychol 27:1-32

30. Benton AL (1968) Differential behavioral effects in frontal lobe disease. Neuropsychologia 6:53-60

31. Houx PJ, Jolles J (1994) Vulnerability factors for age-related cognitive decline. In: Isaacson RL, Jensen KF (Eds) The vulnerable brain and environmental risks. Plenum Press, New York, pp 25-41 